\title{
Gastrosquise: Avaliação Pré-Natal dos Fatores Prognósticos para Sobrevida Pós-Natal
}

\author{
Gastroschisis: Prenatal Evaluation of Prognostic Factors for Postnatal Outcome \\ Liliana Patroni ${ }^{1}$, Maria de Lourdes Brizot ${ }^{1}$, Samir A. Mustafá ${ }^{1}$, Mário H.B. Carvalho ${ }^{1}$, \\ Marcos Marquês Silva², Seyzo Miyadahira ${ }^{1}$, Marcelo Zugaib ${ }^{1}$.
}

\section{RESUMO}

Objetivo: avaliar a evolução de 24 casos de gastrosquise, em relação aos fatores prognósticos pré-natais que interferiram na sobrevida pós-natal.

Pacientes e Métodos: foram avaliados 24 casos de gastrosquise diagnosticados no Setor de Medicina Fetal do Hospital das Clinicas da FMUSP, durante um periodo de 8 anos. Foram classificados em gastrosquise associada, quando presentes outras malformações, e isolada. Nos dois grupos foram analisados parâmetros referentes às alças intestinais dilatadas na avaliação ultra-sonográfica (dilatação $\geq 18 \mathrm{~mm}$ ), complicações obstétricas e resultados pós-natal. Resultados: foram observados 9 casos de gastrosquise associada (37,5\%) e 15 casos de gastrosquise isolada (62,5\%). Todos os casos de gastrosquise associada foram de prognóstico letal, levando a uma alta taxa de mortalidade geral de 60,8\%. Do grupo de gastrosquises isoladas, todos nasceram vivos e foram submetidos a cirurgia, com taxa de sobrevida de $60 \%$ e mortalidade pós-natal de 40\%. A mediana da idade gestacional foi de 35 semanas e o peso no nascimento de 2.365 gramas no grupo geral. Nas gastrosquises isoladas, o parto prematuro ocorreu em 10 casos, principalmente decorrente de complicações obstétricas. Dois recém-nascidos foram considerados pequenos para a idade gestacional e apenas 3 apresentaram peso no nascimento $\geq 2.500$ gramas. O oligoidrâmnio foi um achado comum (46,6\%), sendo mais freqüente no grupo que evoluiu para óbito neonatal $(66,7 \%)$. A avaliação ultra-sonográfica das alças intestinais demonstrou que em 13 de 15 casos (86,6\%) as alças eram dilatadas, mas sem relação significativa com o prognóstico e achados pós-natais. Não houve diferença significativa em relação a idade gestacional e peso no nascimento, comparando os grupos de vivos ébitos neonatais.

Conclusões: as gastrosquises isoladas apresentam um melhor prognóstico quando comparadas às associadas, sendo de suma importância a sua diferenciação pré-natal. As gastrosquises isoladas estão associadas a complicações obstétricas (60\%), prematuridade e baixo peso ao nascimento. O diagnóstico pré-natal permite uma melhor monitorização das condições fetais. $O$ parto destas gestações deve ser no termo, a menos que complicações obstétricas se apresentem.

PALAVRAS-CHAVE: Gastrosquise. Diagnóstico pré-natal. Malformação fetal.

\footnotetext{
${ }^{1}$ Setor de Medicina Fetal da Clínica Obstétrica do Hospital das Clínicas da FMUSP

${ }^{2}$ Disciplina de Cirurgia Pediátrica do Departamento de Pediatria do Instituto da Criança do HCFMUSP.

Correspondência: Maria de Lourdes Brizot

Setor de Medicina Fetal da Clínica Obstétrica do Hospital das Clínicas da FMUSP

Departamento de Obstetrícia, Instituto Central 10o Andar Sala 10083.

Rua Dr. Enéas de Carvalho Aguiar, 255

05403-000 - São Paulo - SP

e-mail: mlbrizot@uol.com.br
}

Introdução

Gastrosquise é uma fenda que interessa toda a espessura da parede abdominal sem envolver o cordão umbilical. Invariavelmente, o defeito é localizado no lado direito do cordão umbilical. Alças intestinais e outros órgãos abdominais podem protruir através desta abertura, sem apresentar membrana peritoneal recobrindo o 
conteúdo exteriorizado, o que a diferencia da onfalocele. A gastrosquise pode ser diagnosticada por meio da ultra-sonografia a partir da $12^{\text {a }}$ semana de gestação ${ }^{1}$. Deve ser diferenciada da onfalocele, uma vez que esta última apresenta uma associação mais freqüente com anomalias cromossômicas e outras malformações, o que modifica a conduta e aconselhamento pré-natal em cada caso.

A gastrosquise é considerada um evento esporádico com etiologia multifatorial. É mais freqüente em gestantes jovens e sua incidência gira em torno de 1 a 2,11 em 10.000 nascidos vivos ${ }^{2}$, e parece não haver predominância de um dos $\operatorname{sexos}^{3,4}$. A predominância em gestantes jovens pode ser secundária ao estilo de vida, relacionado a uma maior exposição a agentes teratogêni$\cos ^{2}$. O defeito é provavelmente devido à isquemia, como resultado da disrupção da artéria onfalomesentérica ou da involução anormal da veia umbilical direita ${ }^{1}$.

$\mathrm{Na}$ gastrosquise isolada o prognóstico é muito bom, com uma taxa de sobrevida pós-correção cirúrgica que pode variar de $43 \%$ a $92,3 \%$ após o diagnóstico pré-natal ${ }^{3,4}$. A sobrevida está diretamente relacionada à presença de outras malformações associadas, presença de complicações pré-natais (oligoidrâmnio, trabalho de parto prematuro, rotura prematura de membranas, sofrimento fetal), prematuridade, peso no nascimento e condições das alças intestinais no nascimento ${ }^{3,5,6}$. O objetivo deste estudo é avaliar a evolução de 24 casos de gastrosquise, em relação aos fatores prognósticos pré-natais que interferiram na sobrevida pós-natal.

\section{Pacientes e Métodos}

Foram avaliados 24 casos de gastrosquise diagnosticados no Setor de Medicina Fetal da Clínica Obstétrica do Hospital das Clínicas da FMUSP, durante o período de março de 1992 a fevereiro de 2000.

Foi considerada gastrosquise a presença de exteriorização do conteúdo abdominal sem membrana envolvendo a herniação, sendo o defeito para-umbilical. As gastrosquises foram classificadas em isoladas, na ausência de outras malformações, e associadas, quando presentes outras malformações.

Os parâmetros avaliados foram: idade materna, idade gestacional quando do diagnóstico ultra-sonográfico, presença e tipo de malformação associada, cariótipo fetal, volume de líquido amniótico (indice de líquido amniótico, normal entre $8 \mathrm{~cm} \mathrm{e} 18 \mathrm{~cm}$ ), idade gestacional no parto e peso no nascimento. Foram verificadas também complicações obstétricas definidas, como: trabalho de parto prematuro, rotura prematura de membranas e sofrimento fetal, o resultado da gestação e o resultado pós-cirúrgico. A ocorrência de crescimento intra-uterino retardado (CIUR) foi definida como peso fetal estimado abaixo do percentil 10 para a idade gestacional.

$\mathrm{O}$ aspecto ultra-sonográfico das alças intestinais foi avaliado em 8 casos de gastrosquise isolada, utilizando-se os critérios de Langer et al. ${ }^{7}$, segundo os quais considera-se alça dilatada quando o diâmetro transverso da alça de intestino delgado for $\geq 18 \mathrm{~mm}$. Para a análise estatística foram calculadas as medianas e variação e, para a comparação entre os grupos, foram utilizados os testes não-paramétricos de MannWhitney e o teste exato de Fisher.

\section{Resultados}

Foram observados 15 casos de gastrosquise isolada e 9 casos de gastrosquise associada a outras malformações. Nos 24 casos a mediana da idade materna foi de 20 anos (16 a 28 anos). A idade gestacional média no diagnóstico foi de 25,2 semanas (16 a 34 semanas). A mortalidade geral nas gastrosquises (isoladas + associadas) foi de $60,8 \%$ e a sobrevida de $39,2 \%$. O cariótipo fetal foi realizado em 8 casos ( 3 com gastrosquise isolada e $5 \mathrm{com}$ malformações associadas), sendo todos os resultados normais. Os recém-nascidos vivos com gastrosquise isolada, sem cariótipo pré-natal, apresentavam o fenótipo normal.

As malformações associadas à gastrosquise (9 de 24 casos, 37,5\%) foram todas graves, sendo que a brida amniótica (4/9) e o defeito extenso de parede abdominal (3/9) foram os mais freqüentes. Nas gastrosquises com malformações associadas, a mediana da idade gestacional no parto foi de 27 semanas (24 a 37) e a mediana do peso no nascimento foi de $1.013 \mathrm{~g}$ (290 a 2.250 g). Neste grupo todos os casos evoluíram para óbito, sendo 5 óbitos intra-uterinos, 3 óbitos neonatais e 1 caso com perda de seguimento.

Nos 15 casos de gastrosquise isolada (15 em 24, 62,5\%), todos nasceram vivos e foram encaminhados para correção cirúrgica (Tabela 1). A mediana da idade gestacional no parto foi de 35 semanas (32 a 38) e a mediana do peso no nascimento foi de 2.365 gramas (1.600 a 3.000). Embora a literatura não demonstre diferença no prognóstico em relação à via de parto nas gestações com gastrosquise ${ }^{8}$, em todos os casos de 
gastrosquise isolada a cesárea foi escolhida, a fim de uma melhor programação conjunta com a equipe da Cirurgia Pediátrica, sendo eletiva a partir da 37a semana. Apenas 4 casos foram para cesárea eletiva na $37^{\text {a }}$ semana e todos evoluíram bem (Tabela 1).

Tabela 1 - Seguimento ultra-sonográfico e evolução pós-natal de 15 casos de gastrosquises isoladas.

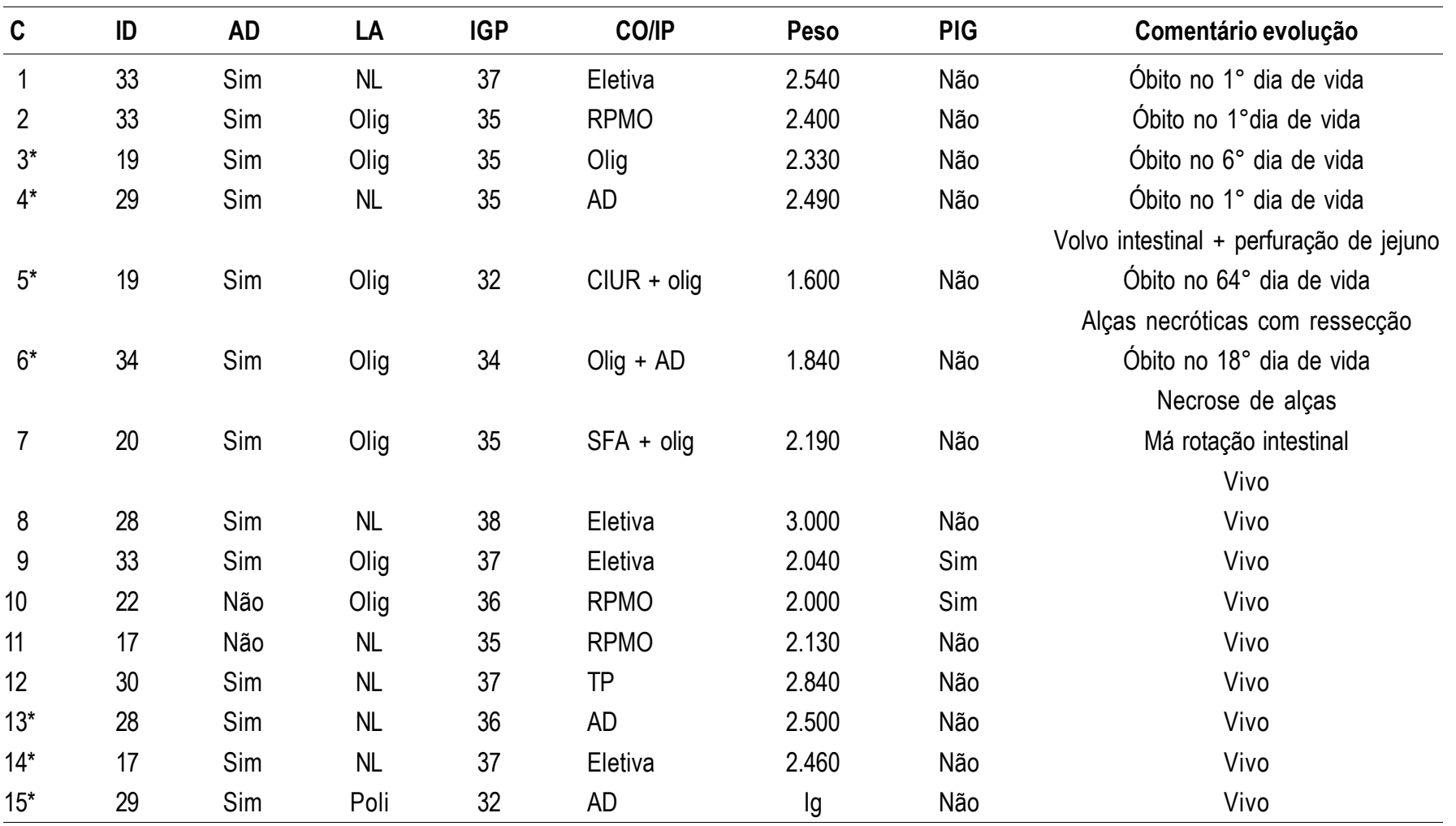

$\mathrm{AD}=$ alças dilatadas no exame ultra-sonográfico; $\mathrm{C}=$ caso; $\mathrm{ClUR}=$ crescimento intra-uterino retardado; $\mathrm{CO} / \mathrm{P}=$ complicações obstétricas; $\mathrm{ID}$ = idade gestacional no diagnóstico ultra-sonográfico; lg = ignorado; IGP = idade gestacional no parto; $\mathrm{LA}$ = líquido amniótico; $\mathrm{NL}$ = normal; Olig = oligoidrâmnio; $\mathrm{PIG}$ = pequeno para a idade gestacional; $P$ Pli = polidrâmnio; $\mathrm{RPMO}=$ rotura prematura de membranas ovulares; SFA = sofrimento fetal agudo; TP = trabalho de parto; "casos anteriores a 1995, quando o critério de interrupp̧ão da gestação considerava o aspecto das alças intestinais e a maturidade pulmonar.

Nas gastrosquises isoladas o parto a termo ocorreu em 5 de 15 casos e apenas 3 destes apresentaram peso $\geq 2.500 \mathrm{~g}$. O parto prematuro $(<37$ semanas) ocorreu em 10 casos e as complicações obstétricas observadas foram: 1 caso com sofrimento fetal agudo e oligoidrâmnio; 3 casos com rotura prematura de membrana, 2 casos com oligoidrâmnio e 1 caso de CIUR e oligoidrâmnio. As complicações obstétricas estiveram presentes em $60 \%(9 / 15)$ de todos os casos (Tabela 1).

Alças dilatadas $(\geq 18 \mathrm{~mm})$ foram observadas em 6 de 8 casos, entretanto, em outros 7 casos foi referida a presença de alças dilatadas ( $\mathrm{n}=13$ ) sem discriminação da medida.

A Tabela 2 compara as características das gastrosquises isoladas do grupo que sobreviveu com o grupo que evoluiu para óbito neonatal. Não foi observada diferença significativa entre os grupos quando avaliados a idade gestacional no parto e o peso no nascimento $(\mathrm{p}=0,12$ e $\mathrm{p}=$ 0,56 , respectivamente).

No grupo das gastrosquises isoladas, o óbito neonatal (ONN) ocorreu em 40\% (6/15) e neste grupo a mediana da idade gestacional no parto foi de 35 semanas (32 a 37) e a mediana do peso no nascimento foi de $2.365 \mathrm{~g}$ (1.600 a $2.540 \mathrm{~g})$. O parto prematuro ocorreu em 5 dos 6 casos $(83,4 \%)$. Houve um caso com diagnóstico de crescimento intra-uterino retardado, não confirmado no pós-natal. Neste grupo todos os recémnascidos foram considerados adequados para a idade gestacional pela curva de Ramos (acima do percentil 10). Dos 6 casos que evoluíram para ONN (apenas 1 de termo), 4 apresentavam alças intestinais dilatadas no exame ultra-sonográfico e a avaliação pós-natal demonstrou: volvo intestinal mais perfuração de jejuno $(n=1)$; alças necróticas $(\mathrm{n}=1)$ e dois casos com alças de aspecto normal. Nos outros dois casos sem dilatação um deles apresentou alças necróticas.

A Tabela 3 apresenta nossos resultados e séries na literatura que avaliam a dilatação das alças intestinais em relação ao prognóstico póscirúrgico. 
Tabela 2 - Características das gastrosquises isoladas de acordo com a evolução: Óbito neonatal e sobreviventes.

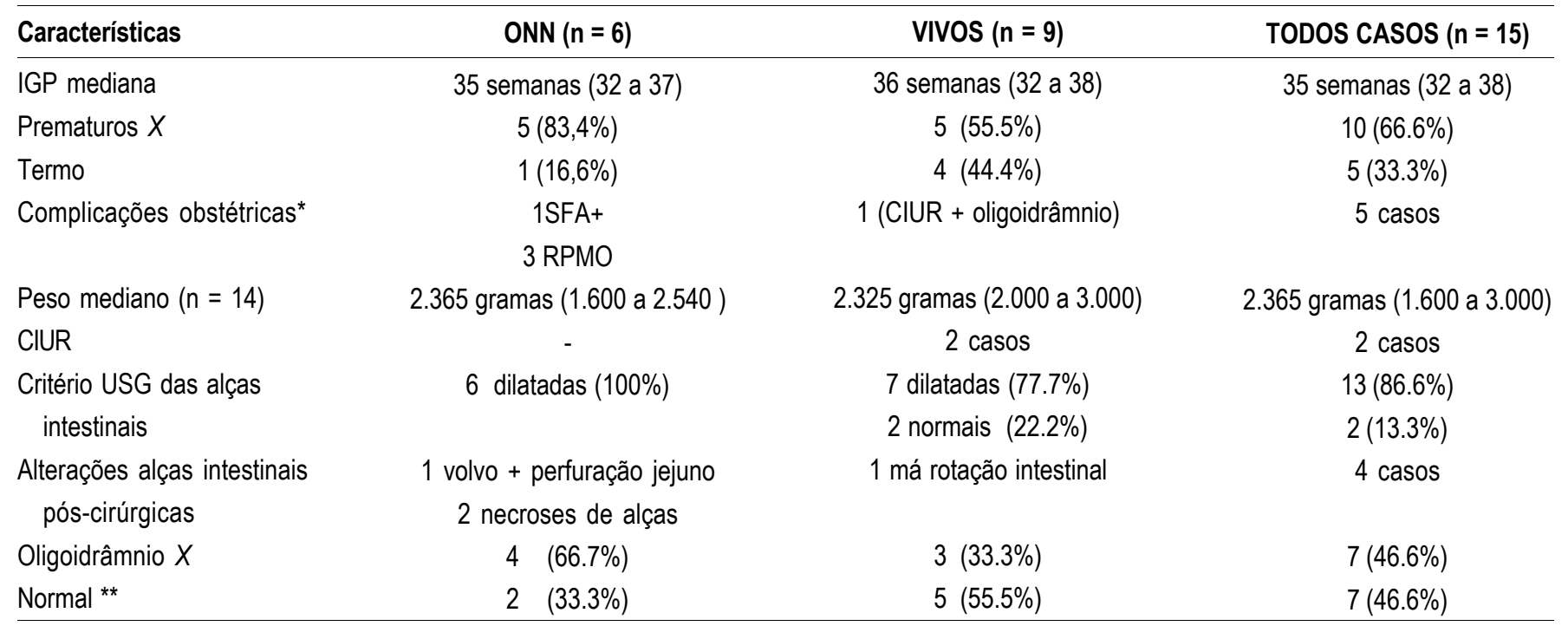

CIUR = crescimento intra-uterino retardado; IGP = idade gestacional no parto; $\mathrm{N}=$ número de casos; $\mathrm{ONN}=$ óbito neonatal; $\mathrm{RPMO}=$ rotura prematura de membranas ovulares; $\mathrm{SFA}=$ sofrimento fetal agudo; USG = ultra-sonográfico; *excluídas as alterações no volume do líquido amniótico. * * um caso de polidrâmnio não foi colocado nesta análise.

Tabela 3 - Séries de gastrosquise na literatura que descrevem o aspecto das alças intestinais e correlacionam com os resultados da gestação.

\begin{tabular}{|c|c|c|c|c|c|c|c|}
\hline NC (AD) & Critério Dilatação & EG & Seguimento Após CC & MM/AD & *Dilatação x Complicação & **Intervenção & Referências \\
\hline $6(3)$ & NM & 6 vivos & 2 óbitos & $3 / 3$ & NM & NM & 16 \\
\hline \multirow[t]{3}{*}{$15(5 / 11)$} & NM & 11 vivos & 1 óbito & $3 / 5$ & Sim & Sim & $19(\mathrm{R})$ \\
\hline & & $3 I T$ & & & & & \\
\hline & & $1 \mathrm{AE}$ & & & & & \\
\hline $5(3)$ & $>10 \mathrm{~mm}$ & NM & NM & $2 / 3$ & Talvez & Não & $18(P)$ \\
\hline $2(2)$ & $>20 \mathrm{~mm}$ & 2 vivos & 0 & $0 / 2$ & Não & Não & $25(P)$ \\
\hline \multirow[t]{2}{*}{$24(6)$} & NM & 21 vivos & 21 vivos & $1 / 6$ & Não & Não & $13(\mathrm{R})$ \\
\hline & & 3 natimorto & & & & & \\
\hline $24(12)$ & $\geq 18 \mathrm{~mm}$ & 24 vivos & NM & $5 / 12$ & Sim & Considerar & $7(\mathrm{R}+\mathrm{P})$ \\
\hline $24(12)$ & $>11 \mathrm{~mm}$ & 24 vivos & 2 óbitos & $7 / 12$ & Sim & Não & $24(\mathrm{R})$ \\
\hline 23 & $>17 \mathrm{~mm}$ & 23 vivos & 3 óbitos & $6 / 23$ & Sim & Considerar & $21(\mathrm{R})$ \\
\hline \multirow[t]{2}{*}{$10(6)$} & NM & 10 vivos & 3 vivos & $3 / 6$ & Não & Não & $3(\mathrm{R})$ \\
\hline & & & 3 óbitos & & & & \\
\hline \multirow[t]{4}{*}{$31(12 / 27)$} & $>10 \mathrm{~mm}$ & 27 vivos & 3 óbitos & $7 / 12$ & Sim & Não & $22(\mathrm{R})$ \\
\hline & & $10 \mathrm{OI}$ & & & & & \\
\hline & & $2 \mathrm{IT}$ & & & & & \\
\hline & & $1 \mathrm{AE}$ & & & & & \\
\hline \multirow[t]{2}{*}{34 (?) } & »17 mm & 34 vivos & 32 vivos & NM & Sim & Considerar & 20 \\
\hline & & & 2 óbitos & & & & \\
\hline $21(8)$ & $\geq 17 \mathrm{~mm}$ & 21 vivos & 0 & $0 / 8$ & Não & Não & $23(\mathrm{R})$ \\
\hline \multirow[t]{2}{*}{19} & NM & 19 vivos & 17 vivos & 1 caso & Não & Não & 9 \\
\hline & & & 2 óbitos & & & & \\
\hline \multirow[t]{2}{*}{$15(13)$} & $\geq 18 \mathrm{~mm}$ & 15 vivos & 9 vivos & $7 / 13$ & Não & Não & Presente \\
\hline & & & 6 ONN & & & & série $(R+P)$ \\
\hline
\end{tabular}

$\mathrm{AD}=$ número de casos com alças dilatadas; $\mathrm{AE}$ = aborto espontâneo; $\mathrm{CC}=$ correção cirúrgica; $\mathrm{EG}$ = evolução da gestação; $\mathrm{IT}$ = interrupção da gestação; $\mathrm{MM} / \mathrm{AD}=$ mortalidade e morbidade/alça dilatada; $\mathrm{NC}=$ número de casos; $\mathrm{NM}=$ não mencionado no texto; $\mathrm{OIU}=$ óbito intra-útero; $\mathrm{ONN}=$ óbito neonatal; $\mathrm{P}=$ análise prospectiva; $\mathrm{R}=$ análise retrospectiva; vivos = nascidos vivos.

* Opinião dos autores sobre se a dilatação de alça intestinal está correlacionada com aumento das complicações cirúrgicas e da morbidade.

** Se os autores recomendam o parto prematuro (<37 semanas), em face do achado ultra-sonográfico de alças dilatadas. 
No grupo com gastrosquise isolada que sobreviveu $(9 / 15,60 \%)$, a mediana da idade gestacional no parto foi de 36 semanas (32 a 38) e a mediana do peso no nascimento foi de $2.325 \mathrm{~g}$ ( 2.000 a $3.000 \mathrm{~g})$. Neste grupo, 2 casos foram considerados pequenos para a idade gestacional e sete casos apresentavam alças dilatadas no exame ultra-sonográfico, sendo 4 pelos critérios de Langer e três por análise subjetiva. Um caso de alça dilatada apresentou má rotação intestinal.

O oligoidrâmnio esteve presente em $46,6 \%$ (7/15), porém, no grupo que evoluiu para ONN observou-se uma maior incidência de oligoidrâmnio $(66,7 \%$ (4/6)) comparado com o grupo de sobreviventes 33,3\% (3/9). Entretanto, não foi observada diferença estatisticamente significativa $(\mathrm{p}=0,755)$ entre os dois grupos. Um caso apresentava polidrâmnio.

Nos 8 casos em que as alças intestinais foram avaliadas segundo os critério de Langer, 6 tinham alças dilatadas $(\geq 18 \mathrm{~mm})$ : dois casos evoluíram para ONN (um com 35 e outro com 37 semanas) e quatro evoluíram bem pós-correção cirúrgica, sendo que um destes apresentou má rotação intestinal e alças edemaciadas. Os dois casos com alças não dilatadas evoluíram bem e sem alteração pós-natal das alças intestinais. Não houve diferença significativa no achado ultra-sonográfico de alças dilatadas em relação ao prognóstico $(\mathrm{p}=0,48)$.

\section{Discussão}

A idade materna média de 20,7 anos, constatada no presente estudo, confere com os dados da literatura, que correlaciona a gastrosquise com baixa idade materna ${ }^{2}$. A importância da diferenciação ultra-sonográfica entre gastrosquise e onfalocele reside no fato de que a gastrosquise raramente se associa a outras malformações e anomalias cromossômicas ${ }^{1}$. Neste estudo, a incidência de malformações associadas foi de $37,5 \%$ e a maioria esteve relacionada à brida amniótica com prognóstico letal. O cariótipo foi normal nos casos em que foi investigado. Outros autores também observaram taxas elevadas de associação com outras anomalias, de $20 \%$ ou mais ${ }^{3}$. Porém, numa análise de 897 casos de gastrosquise, a ocorrência de malformações associadas foi de $6,8 \%(61 / 897)$ e de anomalias cromossômicas de $0,68 \%(61 / 897)^{3,4}$.

A nossa taxa de mortalidade geral $(60,8 \%)$ aumentou em função do mau prognóstico dos fetos com anomalias associadas e é muito maior do que outras séries (10-53\%) nas quais a asso- ciação com outras malformações é menor ${ }^{3}$. No grupo de gastrosquise isolada, a taxa de sobrevida foi de $60 \%$ (9/15). Poulain et al. ${ }^{3}$ também observaram taxa de sobrevida semelhante (57\%). Entretanto, vários outros estudos relatam sobrevida em torno de $85-92,3 \%^{4,9}$. A baixa sobrevida observada nos nossos casos esteve relacionada às complicações obstétricas, como prematuridade, baixo peso e alterações nas alças intestinais no pós-natal.

Fetos com gastrosquise apresentam algumas complicações que resultam em um impacto na taxa de sobrevida neonatal. Uma das complicações obstétricas freqüentes é o CIUR, e a sua associação com gastrosquise ocorreu entre $20 \%$ e $80 \%{ }^{10}$. Raynor e Richards ${ }^{10}$, encontraram $24 \%$ de CIUR, com um peso médio de $2.401 \mathrm{~g}( \pm 508$ g) e peso abaixo de $2.500 \mathrm{~g}$ em $56 \%$ dos casos. O mecanismo do CIUR permanece incerto, podendo ser decorrente de uma deprivação nutricional, secundária a uma perda de nutrientes e proteínas através da parede da alça exposta ao liquido amniótico ${ }^{10}$.

$\mathrm{Na}$ presente série de gastrosquise isolada a mediana do peso no nascimento foi de 2.365 gramas (1.600 a 3.000), não havendo diferença significativa entre os que evoluíram para ONN e aqueles que sobreviveram $(\mathrm{p}=0,56)$. Dois dos 14 fetos $(14,2 \%)$ nasceram com peso abaixo do percentil 10 para a idade gestacional, porém apresentaram boa evolução pós-natal. Um retardo de crescimento moderado pode estar presente em fetos com gastrosquise sem afetar o prognóstico $^{3,11}$. No entanto, recém-nascidos de baixo peso apresentam mais complicações pós-operatórias ${ }^{5}$. Entre os casos com gastrosquise isolada, 10 casos $(71,4 \%)$ apresentaram peso no nascimento $<2.500 \mathrm{~g}$, sendo mais comum no grupo que evoluiu para ONN. Das 5 gestações que evoluíram até o termo, somente 3 apresentaram peso no nascimento $\geq 2.500$ gramas. Blakelock et al. ${ }^{12}$ relatam que fetos nascidos a termo, com gastrosquise, são considerados pequenos para a idade gestacional quando comparados a fetos nascidos prematuros com gastrosquise. Estes autores sugerem que no final da gestação o crescimento fetal adequado é dependente de um funcionamento normal do trato gastrointestinal.

Alterações no volume de líquido amniótico, tanto um aumento quanto uma diminuição, podem estar associadas à gastrosquise ${ }^{13}$. No grupo com gastrosquise isolada, foi observado oligoidrâmnio em $46,6 \%$, sendo mais freqüente nos casos que evoluíram para ONN $(66,6 \%)$, o que sugere ser um fator prognóstico na sobrevida pósnatal ${ }^{5,10}$.

A alta incidência de complicações obstétri- 
cas associadas à gastrosquise indica a maior necessidade de monitorização antenatal, independentemente da presença ou não de complicações como oligoidrâmnio e $\mathrm{CIUR}^{13}$. Em análise de 764 casos, foi observado aproximadamente $1 \%$ de óbito intra-uterino inexplicado nas últimas semanas de gestação e $7,4 \%$ de sofrimento fetal agudo ou neonatal ${ }^{3}$. Crawford et al. ${ }^{13}$ relataram $12,5 \%$ de natimortos no final do terceiro trimestre, portanto, é importante a avaliação semanal ou quinzenal a partir da $30^{\text {a }}$ semana de gestação.

Nas gestações de fetos com gastrosquise, o trabalho de parto prematuro ocorre com freqüência de um terço a $72,7 \%{ }^{1,14}$. A sua etiologia é pouco entendida, podendo ser resultante da ação de mediadores inflamatórios no líquido amniótico ${ }^{9,15}$. No presente estudo, o parto prematuro ocorreu em dois terços (10/15) dos casos de gastrosquise isolada e as complicações obstétricas estiveram presentes em 7 destes.

Embora malformações extra-intestinais sejam raras em associação com gastrosquise, anomalias gastrointestinais estão presentes em 20$40 \%$ dos $\operatorname{casos}^{1}$. Estas anomalias gastrointestinais incluem: má-rotação, atresia intestinal, volvo e infartos, e são estas alterações que apresentam maior impacto no prognóstico pós-natal da gastrosquise. A atresia intestinal e hipoperistaltismo no pós-operatório podem ser explicados pela isquemia e compressão que ocorrem, de acordo com o tamanho do defeito. Foram observadas anomalias do trato gastrointestinal póscirurgia em 4 casos: 1 volvo intestinal com perfuração de jejuno, 2 casos com alças necróticas e um caso de má rotação intestinal.

O prognóstico do recém-nascido com gastrosquise também depende das condições do intestino no nascimento ${ }^{5}$. Vários estudos clínicos e experiências em animais sugerem que o dano intestinal na gastrosquise ocorre antes do parto e, mais provavelmente, nas últimas semanas de gestação ${ }^{16}$. Embora a etiologia do dano intestinal não esteja completamente esclarecida, várias considerações, referentes ao acometimento das alças intestinais, são descritas na literatura. Estudos recentes, dosando mediadores inflamatórios no líquido amniótico, informam que existe uma resposta inflamatória no líquido amniótico de fetos com gastrosquise, que pode levar a uma periviscerite com lesão de alça intestinal $^{9,15}$. Alguns estudos, em modelos animais e em fetos humanos com gastrosquise, demonstraram que uma amnioinfusão ou troca de liquido amniótico poderia melhorar o prognóstico destas gestações, provavelmente pela diminuição da resposta inflamatória ${ }^{15,17}$.
O espessamento das alças intestinais e a formação de camada fibrosa agrupando estas alças foram atribuídas à longa exposição ao líquido amniótico e, especialmente, às mudanças na composição deste líquido, em decorrência da maturidade da função renal ${ }^{16}$. Esta alteração fibrótica parece não ocorrer em alças intestinais de fetos com menos de 30 semanas $^{16}$. Portanto, o prognóstico deveria ser melhor nos casos com parto prematuro, antes da 37a semana de gestação ${ }^{18}$. Bond et al. ${ }^{19}$ não observaram uma correlação positiva entre o tempo de exposição das alças intestinais ao líquido amniótico com o acometimento das alças, e sim com a dilatação e espessamento da parede das mesmas. Estes autores acreditam que a atresia e o infarto de alças intestinais ocorrem tardiamente na gestação e são resultantes do estrangulamento do intestino eviscerado, pois defeitos pequenos e a falta de adesão do intestino ao mesentério contribuiriam para a rotação das alças sobre seu pedículo vascular, levando à lesão das alças.

$\mathrm{O}$ aspecto ultra-sonográfico das alças intestinais foi analisado em vários estudos, tentando-se correlacionar a sua alteração com o prognóstico da gastrosquise (Tabela 3). Alterações ultra-sonográficas das alças intestinais, tais como a dilatação e o espessamento da parede, tiveram correlação positiva com a presença de necrose, atresia intestinal e hipoperistaltismo no recém-nascido ${ }^{7,19,20}$. Langer et al. ${ }^{7}$, avaliando 24 fetos com gastrosquise, em idade gestacional variando de 33 a 38 semanas, observaram que dilatação intestinal maior que $18 \mathrm{~mm}$ correlacionava-se positivamente com necessidade de ressecção intestinal e presença de hipoperistaltismo. Já a espessura da parede intestinal $\geq 4$ $\mathrm{mm}$ não apresentou correlação significativa com aquelas complicações. Outros autores também não observaram uma correlação entre dilatação de alças e piora do prognóstico pós-cirúrgico, entretanto, demonstraram maior incidência de sofrimento fetal durante o trabalho de parto e menor peso no nascimento ${ }^{13}$. No presente estudo não foi observada correlação entre alças com dilatação $\geq 18 \mathrm{~mm}$ e prognóstico pós-cirúrgico ou lesão de alça intestinal. O aspecto ultra-sonográfico das alças intestinais, como indicador do seu comprometimento, era utilizado em nosso serviço como critério para determinar a antecipação do parto, muitas vezes adicionando a prematuridade às complicações cirúrgicas. No entanto, desde 1995 a alteração ultra-sonográfica das alças não é parâmetro indicativo de resolução; a partir desta data passamos a considerar outras variáveis, tais como presença de oligoidrâmnia ou alteração na vitalidade fetal. 
Atualmente, de acordo com a literatura ${ }^{5,6,21}$, nos parece que o melhor momento para a interrupção da gestação é após a 37ạ semana, já que se observa uma menor morbidade pós-cirúrgica em fetos a termo, não se utilizando mais o critério de presença de maturidade pulmonar. A maioria dos autores não recomendam a antecipação do parto $(<37$ semanas) baseada somente na alteração do aspecto ultra-sonográfico das alças intestinais ${ }^{5,6,13,18,21-25}$. A maioria dos estudos são retrospectivos e as casuísticas são pequenas. Avaliações prospectivas e provavelmente multicêntricas serão necessárias para esclarecer melhor os achados dos estudos apresentados até o presente momento (Tabela 3).

O prognóstico dos recém-nascidos com gastrosquise isolada dependeu, principalmente, da idade gestacional no parto. A baixa taxa de sobrevida observada no presente estudo (60\%), quando comparada com a literatura, foi influenciada, principalmente, pela alta taxa de prematuridade (10 casos com idade gestacional inferior a 37 semanas) com baixo peso associada a uma maior incidência de oligoidrâmnia. O diagnóstico pré-natal permite uma melhor monitorização das condições fetais. O parto destas gestações deve ser no termo, a menos que complicações obstétricas se apresentem.

\section{SUMMARY}

Purpose: to evaluate 24 cases of gastroschisis, in relation to the prognostic factors that interfered with postnatal outcome.

Patients and Method: twenty-four pregnancies with fetal prenatal ultrasound diagnosis of gastroschisis, during an 8-year period, were analyzed. Gastroschisis was classified into isolated, when there were no other structural abnormalities, or associated, when other abnormalities were present. For both groups the following parameters were examined: ultrasound bowel dilatation $(\geq 18 \mathrm{~mm})$, obstetric complications and postnatal outcome. Nonparametric Mann-Whitney and exact Fisher's tests were used for statistical analyses.

Results: in 9 cases (37.5\%) gastroschisis was associated with other abnormalities, and in 15 cases it was isolated $(62.5 \%)$. All cases of associated gastroschisis had a letal prognosis, therefore the overall mortality rate was $60.8 \%$. In the group of isolated gastroschisis, all were born alive and were submitted to surgery, but the survival rate after surgical correction was $60 \%$. The median gestational age at birth was 35 weeks and birth weight 2,365 grams. Premature delivery was observed in 10 cases, mainly as a consequence of obstetric complication. Two newborns were small for gestational age, and only 3 had birth weight $\geq 2,500$ grams. Oligohydramnios was found in $46.6 \%$ and it was more frequent in the group of postnatal death (66.7\%). Ultrasound assessment of bowel showed bowel dilatation in $86.6 \%$, however, without relation to the prognosis and postnatal bowel findings. There was no significant difference between gestational age at birth and birth weight comparing the survivor and postnatal death groups.

Conclusions: isolated gastroschisis had a better prognosis when compared to associated, therefore this prenatal differentiation is important. Isolated gastroschisis was often associated with prematurity, small birth weight and obstetric complications. Prenatal diagnosis allows better monitoring of fetal and obstetric conditions. Delivery should be at term, unless presenting with obstetric complications.

KEY WORDS: Gastroschisis. Prenatal diagnosis. Fetal malformation.

\section{Referências}

1. Paidas MJ, Crombleholme TM, Robertson RM. Prenatal diagnosis and management of the fetus with an abdominal wall defect. Semin Perinatol 1994; 18:196-214.

2. Penman DG, Fisher RM, Noblett HR, Soothill PW. Increase in incidence of gastroschisis in the south west of England in 1995. Br J Obstet Gynaecol 1998; 105:328-31.

3. Poulain P, Milon J, Frémont B, et al. Remarks about the prognosis in case of antenatal diagnosis of gastroschisis. Eur J Obstet Gynecol Reprod Biol 1994; 54:185-90.

4. Rankin J, Dillon E, Wright C. Congenital anterior abdominal wall defects in the north of England, 1986-1996: occurrence and outcome. Prenat Diagn 1999;19:662-8.

5. Blakelock RT, Harding JE, Kolbe A, Pease PW. Gastroschisis: can the morbidity be avoided? Pediatr Surg Int 1997; 12:276-82 .

6. Simmons M, Georgeson KE. The effect of gestacional age at birth on morbidity in patients with gastroschisis. J Pediatr Surg 1996; 31:1060-2.

7. Langer JC, Khanna J, Caco C, Dykes EH, Nicolaides KH. Prenatal diagnosis of gastroschisis: development of objective sonographic criteria for predicting outcome. Obstet Gynecol 1993; 81:536.

8. Sbragia Neto L, Melo Filho AA, Barini R, Hughet PR, Marba S, Bustorff-Silva JM. Importância do diagnóstico pré-natal de gastrosquise. Rev Bras Ginecol Obstet 1999; 21:475-9. 
9. Luton D, De Lagausie P, Guibourdenche J, et al. Prognostic factors of prenatally diagnosed gastroschisis. Fetal Diagn Ther 1997; 12:7-14.

10. Raynor BD, Richards D. Growth retardation in fetuses with gastroschisis. J Ultrasound Med 1997; 16:13-6.

11.Adair CD, Rosnes J, Frye AH, Burrus DR, Nelson $\mathrm{LH}$, Veille J-C. The role of antepartum surveillance in the management of gastroschisis. Int J Gynecol Obstet 1996; 52:141-4.

12.Blakelock R, Upadhyay V, Kimble R, Pease P, Kolbe A, Harding $J$. Is a normally functioning gastrointestinal tract necessary for normal growth in late gestation? Pediatr Surg Int 1998; $13: 17-20$

13. Crawford RA, Ryan G, Wright VM, Rodeck CH. The importance of serial biophysical assessment of fetal wellbeing in gastroschisis. $\mathrm{Br} \mathrm{J}$ Obstet Gynaecol 1992; 99:899-902.

14.Heydanus R, Raats MAM, Tibboel D, Los FJ, Wladimiroff JW. Prenatal diagnosis of fetal abdominal wall defects: a retrospective analysis of 44 cases. Prenat Diagn 1996; 16:411-7.

15.Morrison JJ, Klein N, Chitty LS, et al. Intra-amniotic inflammation in human gastroschisis: possible aetiology of postnatal bowel dysfunction. $\mathrm{Br} \mathrm{J}$ Obstet Gynaecol 1998; 105:1200-4.

16.Tibboel D, Raine P, McNee M, et al. Developmental aspects of gastroschisis. J Pediatr Surg 1986; 21:865-9.

17.Luton D, De Lagausie P, Guibourdenche J, et al. Effect of amnioinfusion on the outcome of prenatally diagnosed gastroschisis. Fetal Diagn Ther 1999;14:152-5.
18.Lenke RR, Persutte WH, Nemes J. Ultrasonographic assessment of intestinal damage in fetuses with gastroschisis: is it of clinical value? Am J Obstet Gynecol 1990; 163:995-8.

19.Bond SJ, Harrison MR, Filly RA, Callen PW, Anderson RA, Golbus MS. Severity of intestinal damage in gastroschisis: Correlation with prenatal sonographic findings. J Pediatr Surg 1988; 23:520-5.

20.Brun M, Grignon A, Guibaud L, Garel L, Saint-Vil D. Gastroschisis: are prenatal ultrasonographic findings useful for assessing the prognosis? Pediatr Radiol 1996; 26:723-6.

21.Pryde PG, Bardicef M, Treadwell MC, Klein M, Isada NB, Evans MI. Gastroschisis: can antenatal ultrasound predict infant outcomes? Obstet Gynecol 1994; 84:505-10.

22.Adra AM, Landy HJ, Nahmias J, Gómez-Marín O. The fetus with gastroschisis: impact of route of delivery and prenatal ultrasonography. Am J Obstet Gynecol 1996; 174:540-6.

23.Alsulyman OM, Monteiro H, Ouzounian JG, Barton L, Songster GS, Kovacs BW. Clinical significance of prenatal ultrasonographic intestinal dilatation in fetuses with gastroschisis. Am J Obstet Gynecol 1996; 175:982-4.

24.Babcook CJ, Hedrick MH, Goldstein RB, et al. Gastroschisis: Can sonography of the fetal bowel accurately predict postnatal outcome? J Ultrasound Med 1994; 13:701-6.

25.Sipes SL, Weiner CP, Williamson RA, Pringle KC, Kimura K. Fetal gastroschisis complicated by bowel dilatation: An indication for imminent delivery? Fetal Diagn Ther 1990; 5:100-3.

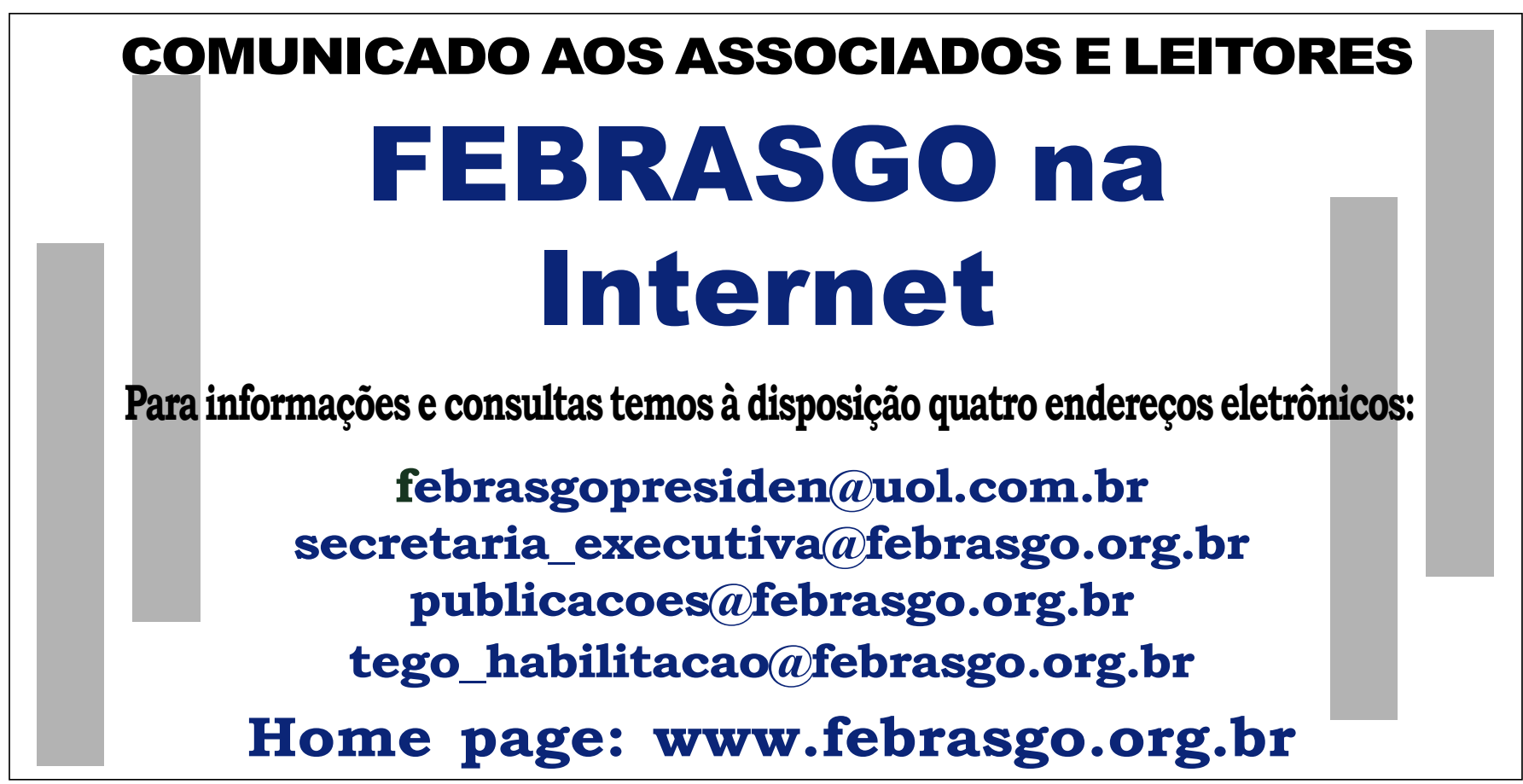

